Received: 2018.10.06

Accepted: 2018.10.30

Published: 2018.11.14

Authors' Contribution: Study Design A

Data Collection B

Statistical Analysis C

Data Interpretation D

Manuscript Preparation E

Literature Search F

Funds Collection $G$

ABCDEF 1,2 Ting Shen

EG 1,2 Qiang Guo

\title{
Role of Pyk2 in Human Cancers
}

1 Faculty of Environmental Science and Engineering, Kunming University of Science and Technology, Kunming, Yunnan, P.R. China

2 Department of Gastroenterology, Affiliated Hospital of Kunming University of

Science and Technology, The First People's Hospital of Yunnan Province, Kunming, Yunnan, P.R. China
Corresponding Author: Source of support:
Qiang Guo, e-mail: gqkj003@sina.com

This study was supported by the National Natural Science Foundation of China (grant No. 81260323) and funding from the First People's Hospital of Yunnan Province, P.R. China (grant No. 2016NS226)

\section{MeSH Keywords: $\quad$ Breast Neoplasms • Cell Migration Assays • Focal Adhesion Kinase 2 • Neoplasm Invasiveness}

Full-text PDF: https://www.medscimonit.com/abstract/index/idArt/913479

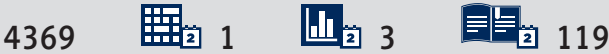

Proline-rich tyrosine kinase 2 (Pyk2) plays essential roles in tumorigenesis and tumor progression. Pyk2 serves as Pyk2 has been traditionally regarded as an oncogene and potential therapeutic target for cancers. However, and focused on regulation of the interconnectivity between Pyk 2 and its downstream targets. The potential use of inhibitors of Pyk2 and its related pathways in cancer therapy is also discussed. 


\section{Background}

Proline-rich tyrosine kinase 2 (Pyk2) is a member of the focal adhesion kinase (FAK) non-receptor tyrosine kinase family and has limited tissue expression, mainly in hematopoietic and neuronal tissues [1,2]. Pyk2 is also known as related adhesion focal tyrosine kinase (RAFTK), cell adhesion kinase $\beta$ (CAK $\beta$ ), and calcium-dependent tyrosine kinase (CADTK), and it can be activated by multiple growth factors (GFs), neuropeptides, cytokines, hormones, and chemokines [2-11]. Pyk2 has been implicated in different signal transduction cascades and plays a critical role in controlling cell adhesion, proliferation, migration, and invasion [12,13]. In recent years, the role that Pyk2 plays in tumor progression has attracted serious attention. Pyk2 has the three-domain organization that is associated with tumor progression (Figure 1), including $\mathrm{NH}_{2}$-terminal FERM domain, centrally located kinase domain, and C-terminal focal adhesion targeting (FAT) domain [14]. The $\mathrm{NH}_{2}$-terminal FERM domain plays a central role in the regulation of Pyk2 kinase activity and receptor association $[15,16]$. Pyk2 FERM domain is composed of 3 structural modules, designated F1, F2, and F3 [17]. F3 module of Pyk2 FERM domain controls tumor cell migration via regulating Pyk2 phosphorylation [16,18-20]. Kinase activity of Pyk2 is generally initiated by autophosphorylation of the tyrosine Y402 situated in the FERM-kinase linker [21]. The phosphorylation sites Tyr402, Tyr579, and Tyr580 are also reported to take part in regulating the growth of tumor cells [22-24]. Pyk2 has the centrally located kinase domain, which may be of potential use in the design of selective kinase inhibitors for targeting cancers $[25,26]$. The C-terminal FAT domain of Pyk2, which has autophosphorylation site Tyr881, is associated with the mitogen-activated protein kinase (MAPK) signaling pathway and cancer development $[26,27]$. In addition, Pyk2 has 3 proline-rich sequences that regulate interactions with proteins. Among them, the C-terminal domain contains 2 proline-rich motifs that control proteins possessing Src homology 3 (SH3) domains [28].

Overexpression of Pyk2 is found in many kinds of malignant tumors and it promotes tumor progression. Pyk2 has traditionally been considered as one of the most promising therapeutic targets for cancer treatment. However, a few studies have found a suppressive role of Pyk2 in tumor growth and metastasis. These paradoxical findings need to be elucidated, as they may be relevant to the adverse effects of Pyk2-targeting treatment and failure of Pyk2-related anti-cancer treatments fail to achieve optimal outcomes. In this review, we provide a new overview of the roles of Pyk2 in multiple cancers. We focus on the interconnectivity between Pyk2 and its downstream targets involved in the biological processes of tumorigenesis and tumor development, as well as furthering the identification of potential targets for cancer therapy and contributing to Pyk2-related anti-cancer drug discovery research.

\section{Aberrant Expression and the Roles of Pyk2 in Different Cancers}

Pyk2 is aberrantly expressed in breast cancer, liver cancer, lung cancer, leukemia, pancreatic cancer, intestinal cancer, multiple myeloma, ovarian cancer, prostate cancer, glioma, squamous cell carcinoma of the head and neck (SCCHN), bladder cancer, and neuroblastoma, compared to normal tissues (Table 1). In most cancers, functional studies have shown that aberrant expression of Pyk2 promotes cancer cell proliferation, migration, invasion, metastasis, and chemo-resistance. However, Pyk2 shows its suppressive role in a few tumors.

\section{Breast Cancer}

Pyk2 is highly expressed and significantly associated with lymph-node metastasis (LNM) in triple-negative breast cancer (TNBC). High levels of Pyk2 and epidermal growth factor receptor (EGFR) contribute to poor prognosis of TNBC patients. Pyk2 depletion can promote proteasomal degradation of tumor-related human epidermal growth factor receptor 3 (HER3), while the Pyk2-NDRG1 ( $\mathrm{N}$-myc downstream regulated 1 gene)NEDD4 (neural precursor cell-expressed developmentally downregulated gene 4) axis is identified as a key regulator of HER3 degradation. Concomitant targeting of EGFR and Pyk2 is proved to inhibit complementary key growth and survival pathways

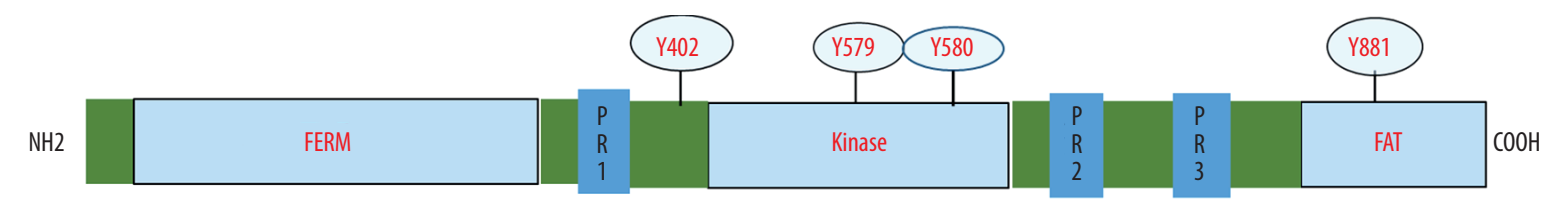

Figure 1. Schematic model of Pyk2 functional domains correlated with tumor progression. Pyk2 contains an N-terminal FERM domain, a central kinase domain, and a C-terminal focal adhesion targeting (FAT) domain. The phosphorylation sites Tyr402, Tyr579, Tyr580, and Tyr881 also take part in regulating the growth of tumor cells. Additionally, Pyk2 includes 3 proline-rich regions located among the structure domains of Pyk2. 
Table 1. The expression and roles of Pyk2 in human cancers.

\begin{tabular}{|c|c|c|c|c|c|}
\hline Cancer type & Incidence & $\begin{array}{l}\text { Expression in } \\
\text { tissues }\end{array}$ & $\begin{array}{c}\text { Downstream target sites of } \\
\text { Pyk2 in cancer }\end{array}$ & $\begin{array}{l}\text { Role of Pyk2 } \\
\text { in cancer }\end{array}$ & Reference \\
\hline Breast cancer & $\begin{array}{l}79.3 \%(73 / 92) \text { of breast } \\
\text { cancer tissue samples is } \\
\text { with high-to-moderate Pyk2 } \\
\text { expression; } 76 \%(41 / 54) \text { of } \\
\text { high-grade breast cancer } \\
\text { tissue samples shows } \\
\text { high Pyk2 expression; } \\
92.8 \%(26 / 28) \text { breast cancer } \\
\text { tissue samples with positive } \\
\text { lymph-node shows high-to- } \\
\text { moderate Pyk2 expression }\end{array}$ & Overexpressed & $\begin{array}{l}\text { p130 Cas; AMAP1; } \beta 1 \\
\text { integrin; c-Met; Twist-1,2; } \\
\text { CD44; fibronectin; Zeb-1,2; } \\
\text { Snail-1,2; ZO-1; MMP; EGFR; } \\
\text { Arg; NDRG1; HER3; } \beta \text {-catenin; } \\
\text { Src; Akt; S6K; vimentin; } \\
\text { E-cadherin; STAT3; ERK }\end{array}$ & Oncogene & $\begin{array}{l}{[12,29,34,36,} \\
38,40,44,119]\end{array}$ \\
\hline Liver cancer & $\begin{array}{l}\text { hepatocellular carcinoma } \\
\text { (59\% or } 29 / 49)\end{array}$ & Overexpressed & $\begin{array}{l}\text { N-cadherin; Hic-5; cytokeratin; } \\
\text { STAT5b; MEK1/2; fibronectin; } \\
\text { Src; Akt; E-cadherin; ERK }\end{array}$ & Oncogene & $\begin{array}{c}{[13,23,46} \\
51,53]\end{array}$ \\
\hline Lung cancer & $\begin{array}{l}97 \%(124 / 128) \text { of NSCLC } \\
\text { patient tissues express Pyk2, } \\
54.7 \%(70 / 128) \text { of NSCLC } \\
\text { patient tissues highly express } \\
\text { Pyk2. 92\% }(118 / 128) \text { of } \\
\text { NSCLC patient tissues express } \\
\text { Pyk2[pY881], 60.2\% (77/128) } \\
\text { of NSCLC patient tissues } \\
\text { highly express Pyk2 [pY881]. }\end{array}$ & Overexpressed & $\begin{array}{l}\text { Src; ALDH1a1; ABCG2; Bmi-1; } \\
\text { ERK }\end{array}$ & Oncogene & {$[5,26,54,57]$} \\
\hline Leukemia & $\begin{array}{l}\text { Pyk2 is expressed in } 81 \% \\
(49 / 60) \text { of } A M L\end{array}$ & $\begin{array}{l}\text { abundantly } \\
\text { express }\end{array}$ & Unknown & Oncogene & {$[67]$} \\
\hline Pancreatic cancer & Unknown & expressed & Unknown & Oncogene & {$[69,71]$} \\
\hline Intestinal cancer & Unknown & Overexpressed & GSK3 $\beta$ & Oncogene & [72] \\
\hline Multiple myeloma & Unknown & Overexpressed & $\begin{array}{l}\text { Paxillin; } \beta \text {-catenin; Src; Akt; } \\
\text { STAT3 }\end{array}$ & Oncogene & {$[73,77]$} \\
\hline Ovarian cancer & $\begin{array}{l}\text { Phosphorylated Pyk2 is } \\
\text { expressed in } 82.1 \%(69 / 84) \text { of } \\
\text { the high-grade serous ovarian } \\
\text { cancer }\end{array}$ & Overexpressed & ERK & Oncogene & {$[7,78,79]$} \\
\hline Prostate cancer & $\begin{array}{l}\text { overexpression of Pyk } 2 \text { is } \\
\text { found in } 32.4 \%(26 / 80) \text { of } \\
\text { prostate cancer tissues }\end{array}$ & expressed & FAK; MAPK; Akt; S6K; ERK & $\begin{array}{l}\text { Oncogene/ } \\
\text { tumor } \\
\text { suppressor }\end{array}$ & $\begin{array}{c}{[81,83,85,} \\
87,92]\end{array}$ \\
\hline Glioma & $\begin{array}{l}\text { Astrocytomas ( } 77.4 \% \text { or } \\
256 / 331 \text { ); glioblastomas } \\
\text { (84.1\% or } 169 / 201 \text { ); other } \\
\text { tumor types of glioma } \\
\text { (unknown) }\end{array}$ & Overexpressed & Rac1; c-Met; ERK & Oncogene & $\begin{array}{c}{[94,98,99} \\
101,108]\end{array}$ \\
\hline SCCHN & Unknown & Overexpressed & Vimentin; E-cadherin; STAT3 & Oncogene & {$[109,112]$} \\
\hline Bladder cancer & Unknown & Overexpressed & Akt; S6K; ERK & Oncogene & [113] \\
\hline Neuroblastoma & Unknown & Unknown & Unknown & $\begin{array}{l}\text { Tumor } \\
\text { suppressor }\end{array}$ & [115] \\
\hline
\end{tabular}


of breast cancer cells, which are mediated by Akt, S6 kinase (S6K), signal transducer and activator of transcription 3 (STAT3), and extracellular signal-regulated protein kinase $1 / 2$ (ERK1/2) activation, and it is a more effective way to overcome HER3associated resistance to EGFR antagonists in TNBC [29]. Pyk2 acts as a crossroads of multiple signaling pathways and promotes breast cancer progression. Carcinogen benzo[a]pyrene diol epoxide (BPDE) increases intracellular $\mathrm{Ca}^{2+}$ concentration and activates the Pyk2/EGFR/Akt signaling pathway, which plays an important role in inhibiting apoptosis of breast cancer cells [30]. Compensatory Pyk2 expression after FAK deletion in mammary cancer stem cells (MaCSCs) promotes breast cancer tumorigenicity and metastasis via activation of the PI3K/ Akt signaling pathway [31]. Glutathione S-transferase omega 1 (GSTO1)-induced cytosolic calcium increase activates the Pyk2/ Src/STAT3 signaling pathway and leads to breast cancer stem cell (BCSC) enrichment, which is essential for breast cancer recurrence and metastasis [32]. Pyk2 promotes ErbB-induced cell proliferation and tumor growth, in part via activating the MAPK signaling pathway [33]. Pyk2 was found to promote migration and invasion of breast cancer cells. Pyk2 is phosphorylated by heregulin (HRG) stimulation and participates in the formation of a multiprotein complex correlated with $\mathrm{p} 190$ RhoGAP (p190), RasGAP, ErbB-2, and Src, and plays a key role in activation of the MAPK signaling pathway and breast cancer cell invasion [34]. Pyk2 is associated with cell adhesion and motility in human breast cancer [35]. Pyk2 promotes breast cancer cell invasion via enhancing matrix metalloproteinase (MMP) secretion, extracellular matrix (ECM) degradation, and invadopodium-mediated functions. Pyk2 colocalizes with cortactin to invadopodia of breast cancer cells, where it regulates epidermal growth factor(EGF)-induced cortactin phosphorylation through Src-mediated Abl-related gene (Arg) activation, resulting in actin polymerization and tumor cell invasion [36]. EGF activates Pyk2 expression, which can promote EMT, migration, invasion, and metastasis of breast cancer cells through modulating the functions of MMP-10, $\beta$-Catenin, fibronectin, vimentin, E-cadherin, ZO-1, Twist-1,2, CD44, Snail-1,2 and Zeb-1,2. With EGF stimulation, Pyk2 affects STAT3 phosphorylation and the protein and mRNA levels of c-Met, c-Met enhances the phosphorylation of Pyk2 and STAT3 and also STAT3 regulates Pyk2 transcription and c-Met expression in human breast carcinoma, forming a positive feedback in the PYK2STAT3-C-Met axis that contributes to cancer metastasis and prolongs EMT-associated signals in breast cancer [12]. Pyk2 $\mathrm{N}$-terminal domain interacting receptor 1 (Nir1) promotes epithelial-mesenchymal transition (EMT) and metastasis of human breast cancer cells by binding to (C-C motif) ligand 18 (CCL18) via activating the Pyk2/Akt/GSK3 $\beta /$ Snail signaling pathway [37]. Transforming growth factor- $\beta$ (TGF- $\beta$ ) upregulates Pyk2 expression, which induces diminished E-cadherin and stabilized $\beta 1$ integrin, facilitates epithelial-mesenchymal transition, and leads to breast cancer cell metastasis, through
Smad4- and Src-dependent pathways [38]. Csk homologous kinase $(\mathrm{CHK})$ is proved to regulate the activation of HRG- and Pyk2-mediated intracellular signaling and breast cancer cell migration [39]. Inhibition of tyrosine phosphorylation of Pyk2 and paxillin can suppress breast cancer cell migration, which is induced by the deficiency of tumor suppressor protein tyrosine phosphatase non-receptor type 12(PTPN12) [40]. Pyk2 plays a key role in chemokine CCL18-induced adhesion, migration, and invasion of breast cancer cells through phosphorylating the GTPase-activating protein AMAP1 [41]. After binding to its functional G protein-coupled receptor PITPNM3, CCL18 in turn activates Pyk2 and Src, which are required for integrin $\alpha / \beta 1$ clustering-dependent cell adherence and cell migration and invasion in breast cancer. Activated Pyk2 translocates from the cytoplasm to the membrane and forms a stable complex with PITPNM3, which activates the intracellular CCL18-induced signaling pathway [42]. C-X-C motif chemokine 12 (CXCL12) can induce the tyrosine phosphorylation of Pyk2 at residues 402 and 579/580, while inhibition of Pyk2 particularly precludes CXCL12-induced cell migration and invasion in breast cancer [43]. The Src/FAK/Pyk2/p130 Cas (crk-associated substrate) pathway is proved to be an effective pathway to control migration and invasion of breast cancer cells [44]. Additionally, inhibition of Pyk2 can arrest estrogen-dependent breast tumor angiogenesis [45]. Alpha-naphthoflavone (ANF) significantly promotes the sensitivity of breast cancer cells to the chemopreventive agent doxorubicin and abrogates doxorubicin resistance via inhibiting phospho-Pyk2 (Y579/580), phospho-FAK (Y397) and EGF-induced Akt activation [22]. Pyk2 acts as an oncogene and enhances tumor progression in breast cancer.

\section{Liver Cancer}

Overexpression of Pyk2 is found in $59 \%$ of hepatocellular carcinoma (HCC) patients. Pyk2 expression is positively correlated with HCC invasiveness, metastasis, recurrence, and poor survival of patients, as well as the gene expression of ezrin and fibronectin [46]. Pyk2 activation leads to cisplatin resistance of hepatocellular carcinoma (HCC) cells through increasing Akt phosphorylation, upregulating drug-resistant genes, and inhibiting cell necrosis and apoptosis [47]. The phosphoinositide 3-kinase (PI3K)/Akt pathway is associated with Pyk2-mediated vascular endothelial growth factor (VEGF) expression, which promotes tumor angiogenesis during HCC progression [48]. Additionally, Pyk2 promotes HCC cell proliferation and invasiveness by activating the c-Src and ERK/MAPK signaling pathways, which can be arrested by overexpression of the Pyk2-related nonkinase (PRNK). The Pyk2 phosphorylated form pY402 plays a role in upregulating actin stress fiber polymerization and HCC cell motility [49]. Pyk2 plays a critical role in liver cancer progression. Overexpression of microRNA 517a (miR-517a) and miR-517c directly decreases Pyk2 expression by specific 
binding to the 3'UTR region (3870-4152) of Pyk2 and cleaving its mRNA. Subsequently, degradation of Pyk2 mRNA inhibits proliferation, migration, and invasion of hepatocellular carcinoma cells [13]. After activating Rho small GTPases Rac1 and RhoA, Pyk2 enhances the formation of membrane ruffles and $\mathrm{HCC}$ cell motility. Pyk2 regulates EMT and promotes HCC progression through downregulating epithelial gene E-cadherin and cytokeratin and upregulating mesenchymal gene hydrogen peroxide inducible clone-5 (Hic-5), STAT5b, Twist, N-cadherin and fibronectin [50]. miR-23b can inhibit EMT of HCC by targeting the 3'UTR region of Pyk2 [51]. Inhibition of receptor membrane-associated phosphatidylinositol transfer protein 3 (PITPNM3) arrests the invasiveness and metastasis of HCC cells through suppressing the activation of Pyk2, which plays a role in supporting the clustering of integrin in HCC [52]. Cytosolic calcium and cytosolic calcium-dependent Pyk2-Src signaling pathway are essential for HBx-regulated HBV DNA replication and reverse transcription, which can support liver cancer development [53]. In cholangiocarcinoma (CC), overexpressed Eph receptor 2 (EphA2) phosphorylates Akt at T308 and Pyk2 at Y402, and at the same time EphA2 activates mammalian target of rapamycin complex1 (mTORC1). The EphA2/Akt/mTORC1 pathway mainly regulates cell proliferation, while the EphA2/ Pyk2/c-Src/ERK pathway plays a major role in modulating CC metastatic ability. Moreover, these 2 pathways also appear to strongly influence each other in CC development [23].

\section{Lung Cancer}

Pyk2 mRNA, total protein, and Pyk2 phosphorylated form pY881 (Pyk2[pY881]) are found to be higher in lung cancer tissues than in normal lung tissues. Pyk2 is proved to be an independent prognostic factor for non-small-cell lung cancer (NSCLC) patients, and patients with high Pyk2 and Pyk2 [pY881] expression show poorer overall survival. Pyk2 is associated with the expression of cancer stem cell markers ALDH1a1, ABCG2, and Bmi-1 [26]. Pyk2 is significantly correlated with advanced stage of NSCLC and lymph-node metastasis. Phosphorylation of Pyk2 can promote the activity of ERK1/2 and result in the progression of NSCLC [54]. The overexpressed Pyk2 in NSCLC is negatively correlated with suppressor cytokine signaling 3 (SOCS3), which can inhibit cell proliferation and invasion in NSCLC [55]. SOCS3 binds to Pyk2 via its Src homology 2 (SH2) and the kinase inhibitory region (KIR) domains, and decreases cell migration of NSCLC through inhibiting Pyk2-associated ERK1/2 activity [56]. Pyk2 acts as a critical downstream effecter in the Src-mediated human lung adenocarcinoma cell survival pathway [57]. Pyk2 and p-Pyk2 are increased significantly in NSCLC apoptotic cells [58]. In small cell lung cancer (SCLC), neuropeptides increase calcium $\left(\mathrm{Ca}^{2+}\right)$ concentration, phosphorylation of highly expressed Pyk2, Src kinase activation, and Pyk2/Src association, which contribute to the GTP-loading of Ras and ERK activation, leading to the promotion of cancer cell proliferation [5].

\section{Leukemia}

Pyk2 is abundantly expressed in hematopoietic cells. In acute promyelocytic leukemia (APL), N-formyl-L-methionyl-L-leucyl-Lphenylalanine (fMLP) induces tyrosine phosphorylation of Pyk2 and $\beta 2$ integrin activation. $\beta 2$ integrin induces cell attachment on fibrinogen-coated dishes, while cell attachment to fibrinogen causes the enhanced Pyk2 phosphorylation. Thus, Pyk2 participates in functional activation of APL cells [59]. Pyk2 plays a critical role in the differentiation of promyelomonocytic leukemic cells, which is induced by stromal cell-derived factor-1 (SDF-1/ CXCL12) and all-trans-retinoic acid (ATRA), accompanied by activation of the transcription factor CCAAT enhancer-binding protein (C/EBP) $\beta$ [60]. After C/EBP $\beta$ binds to and transactivates the Pyk2 promoter, the expression of Pyk2 is upregulated during phorbol 12-mystate 13-acetate (PMA)-induced monocytic differentiation of acute promyelocytic leukemia cells through the MAPK/ERK pathway [61]. PMA treatment upregulates the expression of Pyk2, while Pyk2 phosphorylation increases upon adherence of human leukemic cell to fibronectin and to stromal cells [62]. In acute promyelocytic leukemia, ATRA treatment upregulates Pyk2 mRNA, protein, and phosphorylation levels and enhances APL cell adhesion, migration, and invasion capabilities. Targeting Pyk2 can inhibit APL cell adhesion and migration, and its interaction with paxillin and vinculin reduces the extramedullary relapse (EMR) in APL [63]. In the same way, Pyk2 inhibition reduces EMR of chronic myeloid leukemia (CML) subsequent to imatinib treatment [64]. Pyk2 plays a key role in CML pathogenesis, which is caused by the p210 BCR/ABL oncoprotein [65]. FAK increases expression of Frizzled-4 and phosphorylates Pyk2 on its Y579 residue to enable the required association of Pyk2 with the Wnt5a/ Frizzled-4/Low-density lipoprotein receptor-related proteins 5 (LRP5) endocytosis complex and $\beta$-catenin activation, thereby maintaining the growth of primitive acute myeloid leukemia (AML) cells [66]. Pyk2 regulates AML cell adhesion and enhances AML progression through cross-talking with FMS-like receptor tyrosine kinase 3 (FLT3) and $\beta 1$ integrin [67]. Ethoxy fagaronine has anti-adhesive potential and inhibits leukemic cell survival via reducing Pyk2 phosphorylation on Tyr 579, impairing $\beta 1$ integrin clustering and inhibiting phosphatidylinositol 3-kinase (PI 3-kinase) activity [24]. Pyk2 acts as an oncogene and participates in maintaining leukemia development.

\section{Pancreatic Cancer}

In pancreatic ductal adenocarcinoma (PDA), Pyk2 expression is easily observed. The cell migration of PDA cells and cells that 
comprise the tumor microenvironment is implicated in the catalytic activity of Pyk2 [68]. Discoidin domain receptor 1 (DDR1), which plays a key role in protumorigenic signaling, can be stimulated by collagen through activating Pyk2 and pseudopodium-enriched atypical kinase 1 (PEAK1) in pancreatic cancer cells [69]. A study shows that Pyk2 acts downstream of DDR1 and is phosphorylated in response to collagen I. DDR1 is in a complex with p130 Crk-associated substrate (p130CAS), and this association is induced by collagen I. Rap1 guanosine triphosphatase (Rap1 GTPase), MAPK kinase (MKK)7, and mixed lineage kinases (MLK) 3 are required for the response to collagen I, which leads to the activation of c-Jun N-terminal kinase 1 (JNK1). c-Jun plays a key role in the process by which activated JNK1 upregulates the expression of N-cadherin, which can promote human pancreatic cancer cells growth, invasion, and metastasis [70]. DDR1 and Pyk2 are necessary for pancreatic cancer development. Isoform b of DDR1 regulates collagen I-induced $\mathrm{N}$-cadherin upregulation, which can promote pancreatic cancer progression, and Src homology and collagen homology 1 (Shc1) play a critical role in this process by coupling to Pyk2 and DDR1 [71].

\section{Intestinal cancer}

Pyk2 is overexpressed in intestinal cancer. In vitro and in vivo, elevated Pyk2 is found to function redundantly in regulation of the Wnt/ $\beta$-catenin pathway by phosphorylating GSK3 $\beta^{\text {r216, }}$ which can induce the recruitment of $\beta$-transducin repeatscontaining proteins $(\beta-\operatorname{TrCP})$ and reinforce intestinal tumorigenesis [72].

\section{Multiple Myeloma}

Patients with multiple myeloma ( $M M)$ are proved to present with overexpression of Pyk2 compared with healthy individuals. In vitro and in vivo, Pyk2 plays a tumor-promoting role in MM cell-cycle progression, adhesion ability, and cell proliferation by activating $\mathrm{Wnt} / \mathrm{\beta}$-catenin signaling. Pyk2 protects $\beta$-catenin from GSK3 $\beta$-induced degradation, while inhibition of Pyk 2 results in destabilizing $\beta$-catenin and downregulation of c-Myc and Cyclin D1. Moreover, inhibition of Pyk2 leads to decreased p-Akt, which indicates that Pyk2 may also regulate $\beta$-catenin through modulating the phosphatidylinositol 3-kinase/Akt/GSK3 $\beta$ pathway [73]. The iron chelator deferasirox (DFX) can inhibit Pyk $2 / \beta$-catenin signaling and induce $M M$ cell apoptosis, following a decrease in reactive oxygen species (ROS) production [74]. Pyk2 is a critical mediator of the multiple myeloma cell survival pathway. Pyk2 shows a more malignant phenotype via enhancing JAK1 (janus kinase 1)/ STAT3 signaling linking $\beta 1$ integrin-mediated adhesion and gp130 (interleukin-6 beta receptor). DEP domain-containing
mTOR-interacting protein (DEPTOR), a negative regulator of the mTOR pathway, is a downstream effector of Pyk2 and STAT3 signaling under fibronectin-mediated adhesion and interleukin-6 (IL-6) stimulation, which regulate MM cell growth and proliferation [75]. IL-6 can inhibit the activation of Pyk2 and the apoptosis of MM cells triggered by dexamethasone (Dex) [76]. Protein tyrosine phosphatase SHP2 is proved to mediate this protective effect of IL- 6 . In Dex-treated MM cells, IL-6 induces selective activation of SHP2, and activated SHP2 interacts with Pyk2 through the phosphorylation site Tyr906 in the C-terminal domain of Pyk2, leading to the dephosphorylation of Pyk2 and the inhibition of MM cell apoptosis [77].

\section{Ovarian Cancer}

Phosphorylated Pyk2 was detected in $82.1 \%$ of high-grade serous ovarian cancer (OC) tissues. Women with phosphorylated Pyk2-positive ovarian cancer had a shorter progression-free survival and overall survival than women with phosphorylated Pyk2-negative ovarian cancer. CCL18 and OC ascites, which has CCL18 in it, elicit Pyk2 activation in OC cells, while activated Pyk2 plays a significant role in ovarian cancer cell migration and ovarian cancer progression [7]. Additionally, ovarian cancer cells are able to secrete IL-6, which induces the phosphorylation of Pyk2, leading to chemo-resistance [78]. Exogenous EGF induces telomerase activity and promotes cancer cell survival through activating the Pyk2/ERK $1 / 2$ pathway and targeting Spl and c-Myc binding sites within the core region of the human telomerase reverse transcriptase gene (hTERT) promoter in malignant ovarian epithelial cells [79]. In murine ID8 ovarian carcinoma cells, Pyk2 knockdown induces elevated p53, which is regulated by Pyk2 FERM domain, inhibits ID8 cell proliferation, and leads to $G 1$ cell cycle arrest [80]. Pyk2 acts as an oncogene in ovarian cancer.

\section{Prostate Cancer}

In prostate cancer, although there is no significant correlation between Pyk2 expression and tumor staging, overexpression of Pyk 2 was found in $32.4 \%$ of tumor samples. Pyk2 is significantly correlated with androgen receptor (AR) function and prostate cancer cell growth via activating ribosomal S6K1 [81]. Pyk2 takes part in numerous signaling pathways and promotes prostate cancer progression. Pyk2 plays an important role in controlling the state of cell differentiation and promotes the proliferation of prostatic cancer cells via activating the MAPK signaling pathway [82]. The elevated ErbB-2, through the activation of Pyk2, increases ERK/MAPK activity and enhances the adhesive ability and metastasis of human prostate cancer (PCa) cells [83]. RhoC, one of the Ras-homologous family genes, enhances tumor distant metastasis by sequentially 
phosphorylating Pyk2, FAK, MAPK, and Akt and increases the invasiveness of prostate tumor cells, which is followed by overexpression of MMP2 and MMP9 [84]. Leupaxin promotes the migration of prostate cancer cells via activating Pyk2, c-Src, and Rho GTPase, which can be inhibited by the cytoplasmic protein tyrosine phosphatase-proline-, glutamate-, serine-, and threonine-rich sequence (PTP-PEST) [85]. However, a few studies showed that the expression of Pyk2 was inversely associated with prostate malignancy. Pyk2 can be found in nearly all normal prostate tissues, while high-grade prostate tumors show complete loss of Pyk2 [86]. The full-length androgen receptor-associated protein ARA55, a nuclear receptor coactivator, enhances the androgen receptor (AR) function in a ligand-dependent manner and plays a negative role in the proliferation and aggression of prostate cancers [87-90]. PhosphoPyk2 directly induces ARA55 phosphorylation at tyrosine 43, impairs the coactivator activity of ARA55 to reduce its interaction with $A R$, and controls prostate tumor progression [91]. Pyk2 is involved in olfactory receptor signaling and conveys the anti-proliferative effect in androgen-sensitive prostate cancer cells. Prostate-specific G-protein-coupled receptor 1 (PSGR1), an olfactory receptor, is activated by $\beta$-ionone, which induces an upregulation in cytosolic $\mathrm{Ca}^{2+}$ and an increase of Pyk2 phosphorylation at Ser375. Subsequently, phospho-Pyk2 activates the p38 MAPK signaling pathway, leads to dephosphorylation in tumor-suppressor protein NDRG1 at Ser330, and plays a key role in inhibiting androgen-dependent prostate tumor progression [92]. To the best of our knowledge, most of the prostate tumor types in which Pyk2 functions as a suppressor in the tumor are androgen-dependent. A study showed that androgen-dependent prostate cancer cells have a surfeit of Pyk2, while androgen-independent prostate cancer cells show a shortage of Pyk2 [93]. The decrease of steroid receptor function is followed by the inhibition of Pyk2 expression. In highgrade prostate cancer, the activity of steroid receptors is destroyed and Pyk2 is found to be totally absent [86]. Pyk2 and steroid receptors cooperate in inhibiting prostate tumor progression through different pathways (Figure 2).

\section{Glioma}

Glioma includes astrocytomas, medulloblastomas, and oligodendroglioma. Pyk2 shows a high expression in glioma in vitro and in vivo. Overexpression of Pyk2 is positively associated with malignant grade of astrocytic tumors, and Pyk2 is found to be overexpressed in $77.4 \%$ of astrocytomas. Additionally, Pyk2 overexpression occurs in $84.1 \%$ of glioblastoma, which are the most malignant astrocytoma [94]. Upregulated Pyk2 significantly increases glioma cell migration [95]. Soluble factors, released from microglia, enhance glioma cell migration by increasing the phosphorylation levels of Pyk2 at Tyr 579/580 [96]. Glioma cell migration requires autophosphorylation of Pyk2 Y402 and

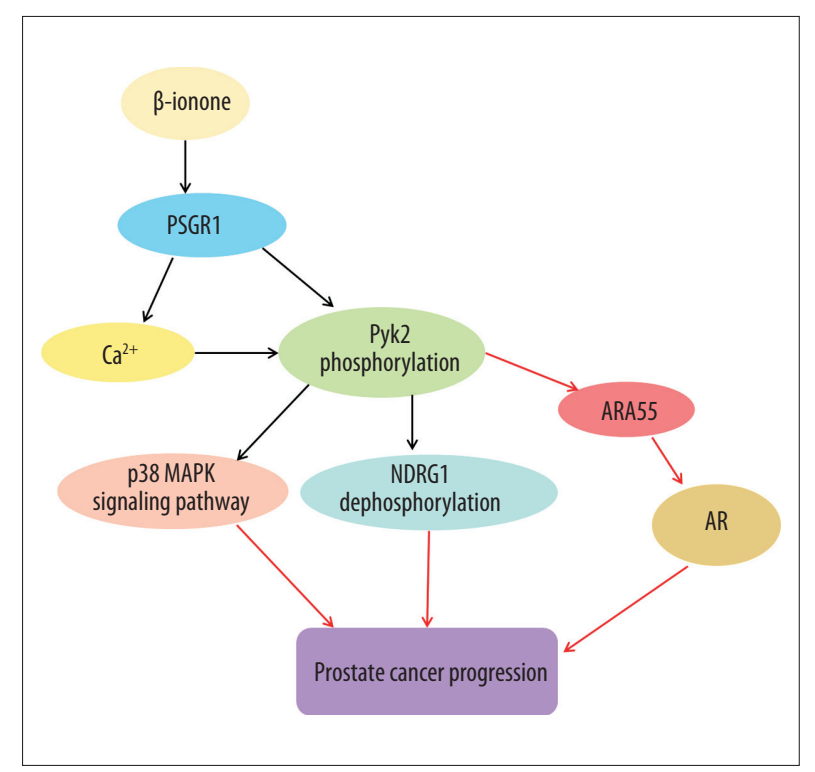

Figure 2. Schematic model of the suppressive role of Pyk2 in prostate cancer progression. Black arrows indicate a promoting effect and red arrows indicate an inhibiting effect. The activation of Pyk2 inhibits cancer progression in androgen-dependent prostate cancer.

the N-terminal FERM domain of Pyk2 [97]. Orai1, the key component mediating Store-operated $\mathrm{Ca}^{2+}$ entry (SOCE), controls glioma cell focal adhesion turnover and epithelial-to-mesenchymal (-like) transition (EMT-like) via the Pyk2 pathway [98]. MiR-23b significantly inhibits glioma cell migration and invasion via targeting the 3' UTR of Pyk2 [99]. Knockdown of Pyk2 inhibits glioma distant metastasis and extends survival duration of orthotopic glioma xenografts [100]. Focal adhesion kinase family interacting protein (FIP200) downregulation enhances the autophosphorylation levels of Pyk2 at Tyr 402, which plays a role in inducing apoptosis of glioblastoma cells [101].

Pyk2 lies downstream of the tumor necrosis factor receptor superfamily expressed on the mouse embryo (TROY), and depletion of Pyk2 suppresses TROY-induced Rac1 activity, followed by inhibition of TROY-mediated glioma cell migration [102,103]. In C6 glioma cells, blockage of $\mathrm{Ca}^{2+}$-permeable nonselective cation channels and inhibition of PI3K attenuate endothelin1-induced Pyk2 phosphorylation [104]. Glioma cell migration and invasion, which is induced by hypoxia, can be decreased by melatonin via inhibiting ROS- $\alpha v \beta 3$ integrin-FAK/Pyk2 signaling pathways [105]. Under the influence of a novel heregulin/HER3-stimulated signaling pathway, phosphorylated Pyk2 activates the MAPK pathway, which plays a critical role in regulating invasiveness of glioma cells [106]. c-Met enhances Pyk2 phosphorylation, while Pyk2 mediates the effects of c-Met on the proliferation, migration, and invasion of medulloblastoma cells [107]. VEGF plays a critical role in tumor development. Although anti-VEGF treatment increases Pyk2 phosphorylation, 
which promotes glioma cell migration and invasion, anti-VEGF treatment plus Pyk2 inhibitor PPI cannot prolong median survival time of rats with intracranial xenograft when compared with anti-VEGF treatment alone [108]. In summary, Pyk2 acts as an oncogene and takes part in many different signaling pathways to promote glioma progression. The roles that Pyk2 play in the different tumor types of glioma are not always the same.

\section{Squamous Cell Carcinoma of the Head and Neck}

Pyk2 is highly upregulated in the squamous cell carcinoma of the head and neck (SCCHN) and metastatic lymph-node cells. Pyk2 inhibitor blunts the phosphorylation of STAT3 elicited by CCL19, which is critical for regulating EMT biology in fibrogenesis and cancer [109]. Additionally, CCL19-induced Pyk2 phosphorylation and cofilin activation are arrested by small GTPase protein RhoA and Rho-associated kinase (ROCK) inhibitors, and this signal pathway is crucial in decreasing SCCHN cell chemotaxis and migration [110]. Pyk2 is a critical modulator of cancer cell migration and invasion. E-cadherin and vimentin may act as downstream target molecules of chemokine receptor 7(CCR7)-Pyk2, and this signaling pathway may participate in the regulation of migration and invasion of squamous cell carcinoma of the head and neck [111]. In squamous cell carcinoma of the head and neck, CCR7 upregulates the phosphorylation of Pyk2 and cofilin activation and enhances cervical lymph-node metastasis, followed by rearrangement of F-actin [110-112].

\section{Bladder Cancer}

Pyk2 is overexpressed in various bladder cancer tissues and mainly locates in the nuclei of urothelial cancer tissue cells. As an oncogene in bladder cancer, Pyk2 serves as a diagnostic and possibly prognostic biomarker. Pyk2 is strongly activated by insulin-like growth factor I (IGF-I) in urothelial carcinoma cells, which is critical for IGF-IR-dependent invasion and can regulate IGF-I-dependent activation of the Akt and MAPK pathways by recruiting insulin receptor substrate-2 (IRS-2) and growth factor receptor-bound protein 2 (Grb2). Knockdown of Pyk2 inhibits IGF-I-dependent activation of ERK1/2 and ribosomal protein S6K, as well as urothelial carcinoma cell growth [113]. Additionally, 2-Arylidenedihydroindole-3-ones decreases bladder tumor cell proliferation via inhibiting the expression of p-Stat5 and p-Pyk2 [114].

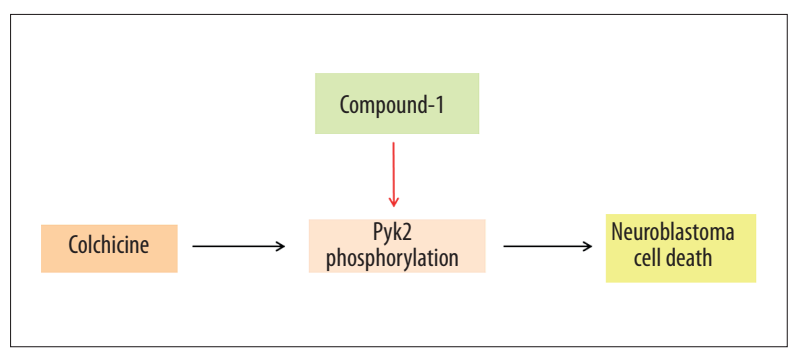

Figure 3. Schematic model of the suppressor role of Pyk2 in neuroblastoma progression. Black arrows show the promoting effect and red arrows show the inhibiting effect. The activation of Pyk2 leads to neuroblastoma cell death. Regulation of Pyk2 phosphorylation can affect neuroblastoma progression.

\section{Neuroblastoma}

The phosphorylation of Pyk2 can be induced by acrylamide, colchicine, and vincristine, while src-family selective tyrosine kinase inhibitor PP1 and compound-1 decrease the phosphorylation of Pyk2 in neuroblastoma cells. Compound-1 plays a role in rescuing colchicine-induced neuroblastoma cell death by inhibiting phosphorylation of Pyk2 (Figure 3). Pyk2 may act as a tumor suppressor in neuroblastoma progression [115].

\section{Other Cancers}

Pyk2 is found to be highly expressed in 34\% of diffuse large $B$ cell lymphoma (DLBCL) patients [116]. In rat pheochromocytoma (PC12) cells, hypoxia induces a strong increase of Pyk2 phosphorylation in the presence of $\mathrm{Ca}^{2+}$. The phospho-Pyk2 then activates MAPK signaling pathways and promotes pheochromocytoma progression [117]. Inhibition of Pyk2 decreases activation of the JNK signaling pathway, which is induced by ultraviolet light or sorbitol, and precludes the growth of PC12 cells [118].

\section{Conclusions}

Pyk2 is widely and highly expressed in human cancers. Numerous correlative studies describing enhanced expression of Pyk2 in human cancers provide strong evidence that Pyk2 serves key roles as oncogenic factors in most of cancers. Pyk2 acts as a supporter and facilities tumor cell proliferation, survival, migration, invasion, metastasis, and chemo-resistance. Overexpression of Pyk2 leads to poor prognosis and shortened survival in various cancers. However, the tumor-suppressive role of Pyk2 has been observed in androgen-dependent prostate cancer cells and neuroblastoma cells. In androgendependent prostate cancers, Pyk2 conveys the anti-proliferative effect and inhibits cancer progression. In neuroblastoma, 
the phosphorylation of Pyk2 results in neuroblastoma cell death. Additionally, activated Pyk2 is observed to induce the apoptosis of lung cancer cells, MM cells, and glioblastoma cells. Most of the prostate tumor types in which Pyk2 exerts suppressive functions in tumors are androgen-dependent. The decrease of steroid receptor function may lead to the inhibition of Pyk2 expression. In high-grade prostate cancer, the damaged steroid receptors are followed by absent of Pyk2. Pyk2 and AR may cooperate on inhibiting prostate cancer progression. Pyk2 has both tumor-driving and tumor-suppressive roles in

\section{References:}

1. Du QS, Ren XR, Xie Y et al: Inhibition of PYK2-induced actin cytoskeleton reorganization, PYK2 autophosphorylation and focal adhesion targeting by FAK. J Cell Sci, 2001; 114(16): 2977-87

2. Avraham S, London R, Fu Y et al: Identification and characterization of a novel related adhesion focal tyrosine kinase (RAFTK) from megakaryocytes and brain. J Biol Chem, 1995; 270(46): 27742-51

3. Di Cioccio V, Strippoli R, Bizzarri C et al: Key role of proline-rich tyrosine kinase 2 in interleukin-8 (CXCL8/IL-8)-mediated human neutrophil chemotaxis. Immunology, 2004; 111(4): 407-15

4. Ivankovic-Dikic I, Gronroos E, Blaukat A et al: Pyk2 and FAK regulate neurite outgrowth induced by growth factors and integrins. Nat Cell Biol, 2000; 2(9): 574-81

5. Roelle S, Grosse R, Buech T et al: Essential role of Pyk2 and Src kinase activation in neuropeptide-induced proliferation of small cell lung cancer cells. Oncogene, 2008; 27(12): 1737-48

6. Cattaneo MG, Lucci G, Vicentini LM: Oxytocin stimulates in vitro angiogen esis via a Pyk-2/Src-dependent mechanism. Exp Cell Res, 2009; 315(18): 3210-19

7. Lane D, Matte I, Laplante C et al: CCL18 from ascites promotes ovarian cancer cell migration through proline-rich tyrosine kinase 2 signaling. Mol Cancer, 2016; 15: 58

8. Schaller MD, Borgman CA, Cobb BS et al: pp125FAK a structurally distinctive protein-tyrosine kinase associated with focal adhesions. Proc Natl Acad Sci USA, 1992; 89(11): 5192-96

9. Lev $\mathrm{S}$, Moreno $\mathrm{H}$, Martinez $\mathrm{R}$ et al: Protein tyrosine kinase PYK2 involved in $\mathrm{Ca}(2+)$-induced regulation of ion channel and MAP kinase functions. Nature, 1995; 376(6543): 737-45

10. Sasaki H, Nagura K, Ishino $M$ et al: Cloning and characterization of cell adhesion kinase beta, a novel protein-tyrosine kinase of the focal adhesion kinase subfamily. J Biol Chem, 1995; 270(36): 21206-19

11. Yu H, Li X, Marchetto GS et al: Activation of a novel calcium-dependent protein-tyrosine kinase. Correlation with c-Jun $\mathrm{N}$-terminal kinase but not mitogen-activated protein kinase activation. J Biol Chem, 1996; 271(47): 29993-98

12. Verma N, Keinan O, Selitrennik M et al: PYK2 sustains endosomal-derived receptor signalling and enhances epithelial-to-mesenchymal transition. Nat Commun, 2015; 6: 6064

13. Liu RF, Xu X, Huang J et al: Down-regulation of miR-517a and miR-517c promotes proliferation of hepatocellular carcinoma cells via targeting Pyk2. Cancer Lett, 2013; 329(2): 164-73

14. Lipinski CA, Loftus JC: Targeting Pyk2 for therapeutic intervention. Expert Opin Ther Targets, 2010; 14(1): 95-108

15. Dunty JM, Schaller MD: The N termini of focal adhesion kinase family members regulate substrate phosphorylation, localization, and cell morphology. J Biol Chem, 2002; 277(47): 45644-54

16. Kohno T, Matsuda E, Sasaki H et al: Protein-tyrosine kinase CAKbeta/PYK2 is activated by binding $\mathrm{Ca}^{2+} /$ calmodulin to FERM F2 alpha2 helix and thus forming its dimer. Biochem J, 2008; 410(3): 513-23

17. Mangeat $P$, Roy C, Martin M: ERM proteins in cell adhesion and membrane dynamics. Trends Cell Biol, 1999; 9(5): 187-92

18. Loftus JC, Yang ZB, Tran NL et al: The Pyk2 FERM domain as a target to inhibit glioma migration. Mol Cancer Ther, 2009; 8(6): 1505-14 cancers in a tissue-dependent manner. Different tumors originate from distinct cell types and grow in diverse environments, which may explain the various Pyk2 responses and contradictory functions of Pyk2 in distinct tumors. The possible adverse effects of promoting cancer progression should be fully considered before treating cancer with Pyk2 inhibitors.

\section{Conflicts of interest}

None.

19. Lipinski CA, Tran NL, Dooley A et al: Critical role of the FERM domain in Pyk2 stimulated glioma cell migration. Biochem Biophys Res Commun, 2006; 349(3): 939-47

20. Meurice N, Wang L, Lipinski CA et al: Structural conservation in band 4.1, ezrin, radixin, moesin (FERM) domains as a guide to identify inhibitors of the proline-rich tyrosine kinase 2. J Med Chem, 2010; 53(2): 669-77

21. Naser R, Aldehaiman A, Diaz-Galicia E et al: Endogenous control mechanisms of FAK and PYK2 and their relevance to cancer development. Cancers (Basel), 2018; 10(6): pii: E196

22. Datta A, Bhasin N, Kim H et al: Selective targeting of FAK-Pyk2 axis by alpha-naphthoflavone abrogates doxorubicin resistance in breast cancer cells. Cancer Lett, 2015; 362(1): 25-35

23. Cui XD, Lee MJ, Kim JH et al: Activation of mammalian target of rapamycin complex 1 (mTORC1) and Raf/Pyk2 by growth factor-mediated Eph receptor 2 (EphA2) is required for cholangiocarcinoma growth and metastasis. Hepatology, 2013; 57(6): 2248-60

24. Ouchani F, Devy J, Rusciani A et al: Targeting focal adhesion assembly by ethoxyfagaronine prevents lymphoblastic cell adhesion to fibronectin. Anal Cell Pathol, 2012; 35(4): 267-84

25. Han S, Mistry A, Chang JS et al: Structural characterization of proline-rich tyrosine kinase 2 (PYK2) reveals a unique (DFG-out) conformation and enables inhibitor design. J Biol Chem, 2009; 284(19): 13193-201

26. Kuang BH, Zhang MQ, Xu LH et al: Proline-rich tyrosine kinase 2 and its phosphorylated form pY881 are novel prognostic markers for non-smallcell lung cancer progression and patients' overall survival. Br J Cancer, 2013; 109(5): 1252-63

27. Blaukat A, Ivankovic-Dikic I, Gronroos E et al: Adaptor proteins Grb2 and Crk couple Pyk2 with activation of specific mitogen-activated protein kinase cascades. J Biol Chem, 1999; 274(21): 14893-901

28. Ren XR, Du QS, Huang YZ et al: Regulation of CDC42 GTPase by prolinerich tyrosine kinase 2 interacting with PSGAP, a novel pleckstrin homology and Src homology 3 domain containing rhoGAP protein. J Cell Biol, 2001; 152(5): 971-83

29. Verma N, Muller AK, Kothari $C$ et al: Targeting of PYK2 synergizes with EGFR antagonists in basal-like TNBC and circumvents HER3-associated resistance via the NEDD4-NDRG1 axis. Cancer Res, 2017; 77(1): 86-99

30. Burdick AD, Ivnitski-Steele ID, Lauer FT et al: PYK2 mediates anti-apoptotic AKT signaling in response to benzo[a]pyrene diol epoxide in mammary epithelial cells. Carcinogenesis, 2006; 27(11): 2331-40

31. Fan HP, Guan JL: Compensatory function of Pyk2 protein in the promotion of focal adhesion kinase (FAK)-null mammary cancer stem cell tumorigenicity and metastatic activity. J Biol Chem, 2011; 286(21): 18573-82

32. Lu HQ, Chen I, Shimoda LA et al: Chemotherapy-induced $\mathrm{Ca}^{2+}$ release stimulates breast cancer stem cell enrichment. Cell Rep, 2017; 18(8): 1946-57

33. Behmoaram E, Bijian K, Jie S et al: Focal adhesion kinase-related prolinerich tyrosine kinase 2 and focal adhesion kinase are co-overexpressed in early-stage and invasive ErbB-2-positive breast cancer and cooperate for breast cancer cell tumorigenesis and invasiveness. Am J Pathol, 2008; 173(5): 1540-50

34. Zrihan-Licht S, Fu YG, Settleman H et al: RAFTK/Pyk2 tyrosine kinase mediates the association of p190 RhoGAP with RasGAP and is involved in breast cancer cell invasion. Oncogene, 2000; 19(10): 1318-28 
35. Rucci N, Ricevuto E, Ficorella C et al: In vivo bone metastases, osteoclastogenic ability, and phenotypic characterization of human cancer cells. Bone, 2004; 34(4): 697-709

36. Genna A, Lapetina S, Lukic N et al: Pyk2 and FAK differentially regulate invadopodia formation and function in breast cancer cells. J Cell Biol, 2018; 217(1): 375-95

37. Zhang BG, Yin CG, Li HL et al: Nir1 promotes invasion of breast cancer cells by binding to chemokine (C-C motif) ligand 18 through the PI3K/Akt/GSK3 beta/Snail signalling pathway. Eur J Cancer, 2013; 49(18): 3900-13

38. Wendt MK, Schiemann BJ, Parvani JG et al: TGF-beta stimulates Pyk2 expression as part of an epithelial-mesenchymal transition program required for metastatic outgrowth of breast cancer. Oncogene, 2013; 32(16): 2005-15

39. McShan GD, Zagozdzon R, Park SY et al: Csk homologous kinase associates with RAFTK/Pyk2 in breast cancer cells and negatively regulates its activation and breast cancer cell migration. Int J Oncol, 2002; 21(1): 197-205

40. Li J, Davidson D, Souza CM et al: Loss of PTPN12 stimulates progression of ErbB2-dependent breast cancer by enhancing cell survival, migration, and epithelial-to-mesenchymal transition. Mol Cell Biol, 2015; 35(23): 4069-82

41. Li HY, Zhang DW, Yu JD et al: CCL18-dependent translocation of AMAP1 is critical for epithelial to mesenchymal transition in breast cancer. J Cell Physiol, 2018; 233(4): 3207-17

42. Li HY, Cui XY, Wu W et al: Pyk2 and Src mediate signaling to CCL18-induced breast cancer metastasis. J Cell Biochem, 2014; 115(3): 596-603

43. Fernandis AZ, Prasad A, Band $\mathrm{H}$ et al: Regulation of CXCR4-mediated chemotaxis and chemoinvasion of breast cancer cells. Oncogene, 2004; 23(1): 157-67

44. Vultur A, Buettner R, Kowolik C et al: SKI-606 (bosutinib), a novel Src kinase inhibitor, suppresses migration and invasion of human breast cancer cells. Mol Cancer Ther, 2008; 7(5): 1185-94

45. Felty Q: Redox sensitive Pyk2 as a target for therapeutics in breast cancer. Front Biosc (Landmark Ed), 2011; 16: 568-77

46. Sun CK, Ng KT, Sun BS et al: The significance of proline-rich tyrosine kinase2 (Pyk2) on hepatocellular carcinoma progression and recurrence. B J Cancer, 2007; 97(1): 50-57

47. Geng W, Ng KT, Sun CK et al: The role of proline rich tyrosine kinase 2 (Pyk2) on cisplatin resistance in hepatocellular carcinoma. PLoS One, 2011; 6(11): e27362

48. Cao J, Chen Y, Fu J et al: High expression of proline-rich tyrosine kinase2 is associated with poor survival of hepatocellular carcinoma via regulating phosphatidylinositol 3-kinase/AKT pathway. Ann Surg Oncol, 2013; 20: S312-23

49. Sun CK, Man K, Ng KT et al: Proline-rich tyrosine kinase 2 (Pyk2) promotes proliferation and invasiveness of hepatocellular carcinoma cells through c-Src/ERK activation. Carcinogenesis, 2008; 29(11): 2096-105

50. Sun CK, Ng KT, Lim ZX et al: Proline-rich tyrosine kinase 2 (Pyk2) promotes cell motility of hepatocellular carcinoma through induction of epithelial to mesenchymal transition. PLoS One, 2011; 6(4): e18878

51. Cao J, Liu JK, Long JY et al: microRNA-23b suppresses epithelial-mesenchymal transition (EMT) and metastasis in hepatocellular carcinoma via targeting Pyk2. Biomed Pharmacother, 2017; 89: 642-50

52. $\mathrm{He} \mathrm{CH}$, Su SC, Chen F et al: Overexpression of PITPNM3 promotes hepatocellular carcinoma cell metastasis. Chinese Science Bulletin, 2014; 59(12) 1326-33

53. Bouchard MJ, Wang LH, Schneider RJ: Calcium signaling by HBx protein in hepatitis B virus DNA replication. Science, 2001; 294(5550): 2376-78

54. Zhang S, Qiu X, Gu Y et al: Up-regulation of proline-rich tyrosine kinase 2 in non-small cell lung cancer. Lung Cancer, 2008; 62(3): 295-301

55. Zhang SY, Wang W, Wang EH et al: SOCS3 expression is inversely correlated with Pyk2 in non-small cell lung cancer and exogenous SOCS3 inhibits proliferation and invasion of A549 cells. Pathology, 2012; 44(5): 434-40

56. Zhang SY, Guo DW, Jiang LL et al: SOCS3 inhibiting migration of A549 cells correlates with PYK2 signaling in vitro. BMC Cancer, 2008; 8: 150

57. Wei L, Yang Y, Zhang X et al: Altered regulation of Src upon cell detachment protects human lung adenocarcinoma cells from anoikis. Oncogene, 2004; 23(56): 9052-61

58. Forest V, Campos L, Vergnon JM et al: Characterization of the focal adhesion complex in human non-small cell lung cancer cell lines. Anticancer Res, 2005; 25(6B): 4135-39

59. Miura Y, Tohyama Y, Hishita T et al: Pyk2 and Syk participate in functional activation of granulocytic HL-60 cells in a different manner. Blood, 2000; 96(5): 1733-39
60. Kim K, Kim HH, Kim JH et al: Chemokine stromal cell-derived factor-1 induction by C/EBP beta activation is associated with all-trans-retinoic acidinduced leukemic cell differentiation. J Leukoc Biol, 2007; 82(5): 1332-39

61. Park MH, Park SY, Kim Y: Induction of proline-rich tyrosine kinase2 (Pyk2) through C/EBP beta is involved in PMA-induced monocyte differentiation. FEBS Lett, 2008; 582(3): 415-22

62. Jeschke M, Brandi ML, Susa M: Expression of SRC family kinases and their putative substrates in the human preosteoclastic cell line FLG 29.1. J Bone Miner Res, 1998; 13(12): 1880-89

63. Ovcharenko A, Granot G, Shpilberg 0 et al: Retinoic acid induces adhesion and migration in NB4 cells through Pyk2 signaling. Leuk Res, 2013; 37(8): 956-62

64. Ovcharenko A, Granot G, Rokah $\mathrm{OH}$ et al: Enhanced adhesion/migration and induction of Pyk2 expression in $\mathrm{K} 562$ cells following imatinib exposure. Leuk Res, 2013; 37(12): 1729-36

65. Salesse S, Dylla SJ, Verfaillie C: p210(BCR/ABL)-induced alteration of premRNA splicing in primary human CD34(+) hematopoietic progenitor cells Leukemia, 2004; 18(4): 727-33

66. Despeaux M, Chicanne G, Rouer E et al: Focal adhesion kinase splice variants maintain primitive acute myeloid leukemia cells through altered Wnt signaling. Stem Cells, 2012; 30(8): 1597-610

67. Katsumi A, Kiyoi H, Abe $A$ et al: FLT3/ITD regulates leukaemia cell adhesion through alpha4beta1 integrin and Pyk2 signalling. Eur J Haematol, 2011; 86(3): 191-98

68. Stokes JB, Adair SJ, Slack-Davis JK et al: Inhibition of focal adhesion kinase by pf-562,271 inhibits the growth and metastasis of pancreatic cancer concomitant with altering the tumor microenvironment. Mol Cancer Ther, 2011; 10(11): 2135-45

69. Aguilera KY, Huang HC, Du WT et al: Inhibition of discoidin domain receptor 1 reduces collagen-mediated tumorigenicity in pancreatic ductal adenocarcinoma. Mol Cancer Ther, 2017; 16(11): 2473-85

70. Shintani Y, Fukumoto Y, Chaika $\mathrm{N}$ et al: Collagen I-mediated up-regulation of $\mathrm{N}$-cadherin requires cooperative signals from integrins and discoidin domain receptor 1. J Cell Biol, 2008; 180(6): 1277-89

71. Huang HC, Svoboda RA, Lazenby AJ et al: Up-regulation of N-cadherin by Collagen I-activated discoidin domain receptor 1 in pancreatic cancer requires the adaptor molecule Shc1. J Biol Chem, 2016; 291(44): 23208-23

72. Gao C, Chen G, Kuan SF et al: FAK/PYK2 promotes the Wnt/beta-catenin pathway and intestinal tumorigenesis by phosphorylating GSK3beta. Elife, 2015; 4

73. Zhang $Y$, Moschetta $M$, Huynh D et al: Pyk2 promotes tumor progression in multiple myeloma. Blood, 2014; 124(17): 2675-86

74. Kamihara Y, Takada K, Sato $T$ et al: The iron chelator deferasirox induces apoptosis by targeting oncogenic Pyk2/beta-catenin signaling in human multiple myeloma. Oncotarget, 2016; 7(39): 64330-41

75. Meads MB, Fang B, Mathews L et al: Targeting PYK2 mediates microenvironment-specific cell death in multiple myeloma. Oncogene, 2016; 35(21) 2723-34

76. Chauhan D, Hideshima T, Pandey P et al: RAFTK/PYK2-dependent and -independent apoptosis in multiple myeloma cells. Oncogene, 1999; 18(48): 6733-40

77. Chauhan D, Pandey P, Hideshima T et al: SHP2 mediates the protective ef fect of interleukin-6 against dexamethasone-induced apoptosis in multiple myeloma cells. J Biol Chem, 2000; 275(36): 27845-50

78. Pasquier J, Gosset M, Geyl C et al: CCL2/CCL5 secreted by the stroma induce IL-6/PYK2 dependent chemoresistance in ovarian cancer. Mol Cancer, 2018; 17: 47

79. Bermudez $\mathrm{Y}$, Yang $\mathrm{H}$, Cheng JQ et al: Pyk2/ERK $1 / 2$ mediate Spl- and c Myc-dependent induction of telomerase activity by epidermal growth factor. Growth Factors, 2008; 26(1): 1-11

80. Lim ST, Miller NLG, Nam JO et al: Pyk2 Inhibition of p53 as an adaptive and intrinsic mechanism facilitating cell proliferation and survival. J Biol Chem, 2010; 285(3): 1743-53

81. Hsiao YH, Huang YT, Hung CY et al: PYK2 via S6K1 regulates the function of androgen receptors and the growth of prostate cancer cells. Endocr Relat Cancer, 2016; 23(8): 651-63

82. Picascia A, Stanzione R, Chieffi P et al: Proline-rich tyrosine kinase 2 regulates proliferation and differentiation of prostate cells. Mol Cell Endocrinol, 2002; 186(1): 81-87 
83. Yuan TC, Lin FF, Veeramani S et al: ErbB-2 via PYK2 upregulates the adhesive ability of androgen receptor-positive human prostate cancer cells. Oncogene, 2007; 26(54): 7552-59

84. liizumi M, Bandyopadhyay S, Pai SK et al: RhoC promotes metastasis via activation of the Pyk2 pathway in prostate cancer. Cancer Res, 2008; 68(18): 7613-20

85. Sahu SN, Nunez S, Bai G et al: Interaction of Pyk2 and PTP-PEST with leupaxin in prostate cancer cells. Am J Physiol Cell Physiol, 2007; 292(6): C2288-96

86. Stanzione R, Picascia A, Chieffi P et al: Variations of proline-rich kinase Pyk2 expression correlate with prostate cancer progression. Lab Invest, 2001; 81(1): 51-59

87. Shibanuma M, Mashimo J, Kuroki T et al: Characterization of the TGF beta 1 -inducible hic- 5 gene that encodes a putative novel zinc finger protein and its possible involvement in cellular senescence. J Biol Chem, 1994; 269(43): 26767-74

88. Heitzer MD, DeFranco DB: Mechanism of action of Hic-5/androgen receptor activator 55, a LIM domain-containing nuclear receptor coactivator. $\mathrm{Mo}$ Endocrinol, 2006; 20(1): 56-64

89. Fujimoto N, Yeh SY, Kang HY et al: Cloning and characterization of androgen receptor coactivator, ARA55, in human prostate. J Biol Chem, 1999; 274(12): 8316-21

90. Miyoshi $\mathrm{Y}$, Ishiguro $\mathrm{H}$, Uemura $\mathrm{H}$ et al: Expression of AR associated protein 55 (ARA55) and androgen receptor in prostate cancer. Prostate, 2003; 56(4): 280-86

91. Wang X, Yang Y, Guo XJ et al: Suppression of androgen receptor transactivation by Pyk2 via interaction and phosphorylation of the ARA55 coregulator. J Biol Chem, 2002; 277(18): 15426-31

92. Wiese H, Gelis L, Wiese $\mathrm{S}$ et al: Quantitative phosphoproteomics reveals the protein tyrosine kinase Pyk2 as a central effector of olfactory receptor signaling in prostate cancer cells. Biochim Biophys Acta, 2015; 1854(6): 632-40

93. Maudsley S, Davidson L, Pawson AJ et al: Gonadotropin-releasing hormone functionally antagonizes testosterone activation of the human androgen receptor in prostate cells through focal adhesion complexes involving Hic5. Neuroendocrinology. 2006; 84(5): 285-300

94. Gutenberg A, Bruck W, Buchfelder M et al: Expression of tyrosine kinases FAK and Pyk2 in 331 human astrocytomas. Acta Neuropathol, 2004; 108(3): 224-30

95. Lipinski CA, Tran NL, Bay C et al: Differential role of proline-rich tyrosine kinase 2 and focal adhesion kinase in determining glioblastoma migration and proliferation. Mol Cancer Res, 2003; 1(5): 323-32

96. Rolon-Reyes K, Kucheryavykh YV, Cubano LA et al: Microglia activate migration of glioma cells through a Pyk2 intracellular pathway. PLoS One, 2015; 10(6): e0131059

97. Lipinski CA, Tran NL, Menashi E et al: The tyrosine kinase Pyk2 promotes migration and invasion of glioma cells. Neoplasia, 2005; 7(5): 435-45

98. Zhu M, Chen L, Zhao P et al: Store-operated $\mathrm{Ca}(2+)$ entry regulates glioma cell migration and invasion via modulation of Pyk2 phosphorylation. J Exp Clin Cancer Res, 2014; 33: 98

99. Loftus JC, Ross JTD, Paquette KM et al: miRNA expression profiling in migrating glioblastoma cells: Regulation of cell migration and invasion by miR-23b via targeting of Pyk2. PLoS One, 2012; 7(6): e39818

100. Lipinski CA, Tran NL, Viso $C$ et al: Extended survival of Pyk2 or FAK deficient orthotopic glioma xenografts. J Neurooncol, 2008; 90(2): 181-89
101. Wang DY, Olman MA, Stewart J et al: Downregulation of FIP200 induces apoptosis of glioblastoma cells and microvascular endothelial cells by enhancing Pyk2 activity. PLoS One, 2011; 6(5): e19629

102. Paulino VM, Yang ZB, Kloss J et al: TROY (TNFRSF19) is overexpressed in advanced glial tumors and promotes glioblastoma cell invasion via Pyk2Rac1 signaling. Mol Cancer Res, 2010; 8(11): 1558-67

103. Jacobs VL, Liu YN, De Leo JA: Propentofylline targets TROY, a novel microglial signaling pathway. PLoS One, 2012; 7(5): e37955

104. Kawanabe Y, Hashimoto N, Masaki T: Effects of nonselective cation channels and PI3K on endothelin-1-induced PYK2 tyrosine phosphorylation in C6 glioma cells. Am J Physiol Cell Physiol, 2003; 285(3): C539-45

105. Xu CS, Wang ZF, Huang XD et al: Involvement of ROS-alpha v beta 3 integrin-FAK/Pyk2 in the inhibitory effect of melatonin on U251 glioma cell migration and invasion under hypoxia. J Transl Med, 2015; 13: 95

106. van der Horst EH, Weber I, Ullrich A: Tyrosine phosphorylation of PYK2 mediates heregulin-induced glioma invasion: Novel heregulin/HER3-stimulated signaling pathway in glioma. Int J Cancer, 2005; 113(5): 689-98

107. Guessous F, Yang Y, Johnson E et al: Cooperation between c-Met and focal adhesion kinase family members in medulloblastoma and implications for therapy. Mol Cancer Ther, 2012; 11(2): 288-97

108. Xu CS, Wang ZF, Dai LM et al: Induction of proline-rich tyrosine kinase 2 activation-mediated $\mathrm{C} 6$ glioma cell invasion after anti-vascular endothelial growth factor therapy. J Transl Med, 2014; 12: 148

109. Liu FY, Safdar J, Li ZN et al: CCR7 regulates cell migration and invasion through JAK2/STAT3 in metastatic squamous cell carcinoma of the head and neck. Biomed Res Int, 2014; 2014: 415375

110. Xu Z, Zheng X, Yang L et al: Chemokine receptor 7 promotes tumor migration and invasiveness via the RhoA/ROCK pathway in metastatic squamous cell carcinoma of the head and neck. Oncol Rep, 2015; 33(2): 849-55

111. Yue Y, Li ZN, Fang QG et al: The role of Pyk2 in the CCR7-mediated regulation of metastasis and viability in squamous cell carcinoma of the head and neck cells in vivo and in vitro. Oncol Rep, 2015; 34(6): 3280-87

112. Yang L, Liu F, Xu Z et al: Chemokine receptor 7 via proline-rich tyrosine kinase- 2 upregulates the chemotaxis and migration ability of squamous cell carcinoma of the head and neck. Oncol Rep, 2012; 28(5): 1659-64

113. Genua M, Xu SQ, Buraschi S et al: Proline-rich tyrosine kinase 2 (Pyk2) reg ulates IGF-I-induced cell motility and invasion of urothelial carcinoma cells. PLoS One, 2012; 7(6): e40148

114. Gerby B, Boumendjel A, Blanc $M$ et al: 2-Arylidenedihydroindole-3-ones: Design, synthesis, and biological activity on bladder carcinoma cell lines. Bioorg Med Chem Lett, 2007; 17(1): 208-13

115. Nakagawa-Yagi Y, Choi DK, Ogane $\mathrm{N}$ et al: Discovery of a novel compound insight into mechanisms for acrylamide-induced axonopathy and colchicine-induced apoptotic neuronal cell death. Brain Res, 2001; 909(1-2): 8-19

116. Bosch R, Dieguez-Gonzalez R, Moreno MJ et al: Focal adhesion protein expression in human diffuse large B-cell lymphoma. Histopathology, 2014 65(1): 119-31

117. Beitner-Johnson D, Ferguson T, Rust RT et al: Calcium-dependent activation of Pyk2 by hypoxia. Cell Signal, 2002; 14(2): 133-37

118. Tokiwa G, Dikic I, Lev $S$ et al: Activation of Pyk2 by stress signals and coupling with JNK signaling pathway. Science, 1996; 273(5276): 792-94

119. Zhang B, Yin C, Li H et al: Nir1 promotes invasion of breast cancer cells by binding to chemokine (C-C motif) ligand 18 through the PI3K/Akt/GSK3beta/ Snail signalling pathway. Eur J Cancer, 2013; 49(18): 3900-13 